\title{
Pflegewissenschaften, (Pflege-)management und HeilberufeSCIENCE
}

\section{J. Klewer}

Online publiziert: 23. Juli 2015

(C) Springer-Verlag Wien 2015

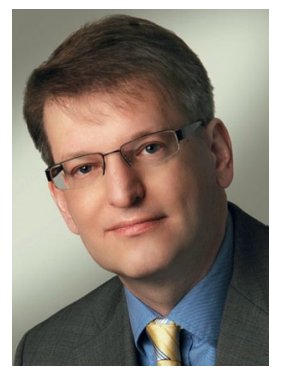

J. Klewer

Wie schon mehrfach an dieser Stelle berichtet, erfordert die kritische Auseinandersetzung mit der eigenen Zeitschrift auch die regelmäßige Analyse des Themenspektrums der veröffentlichten Artikel. Dabei zeigt sich auch im siebten Jahr von HeilberufeSCIENCE, dass der inhaltliche Schwerpunkt der meisten Artikel weiterhin auf den Gebieten Pflegewissenschaften und (Pflege-)management liegt, sowohl in Krankenhäusern als auch stationären Pflegeeinrichtungen, wobei thematische Überschneidungen innerhalb eines Beitrags möglich sind. Artikel mit pflegepädagogischen sowie Public Health-bezogenen Themenstellungen wurden bislang deutlich seltener eingereicht. Sicherlich haben sowohl Pflegepädagogik als auch Public Health einen inhaltlichen Bezug zu Pflegewissenschaften und (Pflege-)management, dennoch möchte ich an dieser Stelle noch einmal dazu einladen, auch Manuskripte zu pflegepädagogischen Studien bzw. Public Health einzureichen, gerne auch als wissenschaftliche Kurzmitteilung.

In dieser Ausgabe von HeilberufeSCIENCE präsentieren wir Ihnen zwei Artikel und zwei wissenschaftliche Kurzmitteilungen, die sich thematisch um das Thema Kundenzufriedenheit bzw. Kundenorientierung gruppieren lassen. Kocksch

J. Klewer $(\bowtie)$

Zwickau, Deutschland

E-Mail: heilberufescience@springer.com und Ullrich untersuchten die Kundenorientierung von Versandapotheken mit einem Onlineshop. Dabei zeigte sich, dass bei diesem immer wichtiger werdenden Marktsegment noch Verbesserungspotenziale existieren, um besser auf die Kundenerwartungen und Kundenbedürfnisse einzugehen. MeyerKühling et al. präsentieren die Ergebnisse einer Untersuchung zu Erwartungshaltungen, Kommunikation und Kooperation von Pflegenden und Ärzten in der stationären Altenpflege. Die Studie zeigt die unterschiedliche Wahrnehmung von Problemen und positiven Aspekten bei der Zusammenarbeit von Pflegenden und Ärzten und schlägt Ansatzpunkte für Interventionsmaßnahmen vor. Giese geht in ihrer wissenschaftlichen Kurzmitteilung darauf ein, wie Patientinnen in Deutschland die Übertragung heilkundlicher Tätigkeiten auf Gesundheits- und Krankenpfleger gemäß $\S 63$, Abs. 3c SGB V bewerten und bietet Ansätze für die diesbezügliche politische Diskussion. In der wissenschaftlichen Kurzmitteilung von Müller et al. steht eine Analyse der Verpflegungssituation von Kindergartenkindern einer Kindertagesstätte im Mittelpunkt. Dabei zeigt sich, dass der gewählte methodische Ansatz zur Evaluation der Verpflegungssituation geeignet ist und dass außerdem noch Optimierungsbedarf bei der Verpflegung der Kleinkinder besteht.

An dieser Stelle möchte ich Sie wieder einladen, die vier Beiträge dieser HeilberufeSCIENCE-Ausgabe kritisch zu lesen und in den wissenschaftlichen Dialog einzutreten. Nutzen Sie dazu auch den 13. Gesundheitspflege-Kongress vom 9. bis 10. Oktober 2015 in Hamburg, um sich über weitere aktuelle pflegewissenschaftliche und gesundheitswissenschaftliche Themen zu informieren. Bis dahin wünsche ich Ihnen eine schöne Sommerzeit.

Ihr

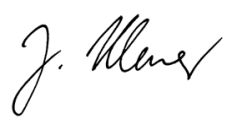

J. Klewer 